\title{
Evaluation of the dry weight rank method for botanical analysis of grassland by means of simulation
}

\author{
J.H. NEUTEBOOM ${ }^{1 *}$, E.A. LANTINGA ${ }^{2}$ AND P.C. STRUIK ${ }^{1}$ \\ 1 Sub-department of Agronomy, Department of Plant and Crop Sciences, Wageningen \\ Agricultural University, Haarweg 333, NL-6709 RZ Wageningen, The Netherlands. \\ ${ }^{2}$ Laboratory of Theoretical Production Ecology, Department of Plant and Crop Sciences, \\ Wageningen Agricultural University, P.O. Box 430, NL-6700 AK Wageningen, The \\ Netherlands. \\ * Corresponding author (fax: +31-317-484575; e-mail: jan.neuteboom@users.agro.wau.nl)
}

Received 22 August 1997; accepted 20 September 1998

\begin{abstract}
With the Dry Weight Rank (DWR) method of 't Mannetje \& Haydock (1963) for botanical analysis in pastures, the dry weight proportions of species are estimated from their first, second and third ranks in dry weight in single quadrats. The yield correction of Haydock \& Shaw (1975) is used additionally to solve the problem of the respective under- and overestimations of the dry weight proportions of high and low yielding species when these grow in patches. In this paper the DWR method is evaluated by means of computer simulation.

Main element of the simulation model is a computer sampling program with which a fictitious vegetation can be sampled with a circular quadrat. The output shows that the DWR method works well using relatively small sampling quadrats with, on average, only a few plants per quadrat, irrespective of the horizontal vegetation structure. In vegetations where species grow patchwise, satisfactory results are also obtained using large quadrats with much more plants (i.e. tens) per quadrat. The reason is that in these cases also minor species can compete successfully for first, second and third ranks. However, it appeared that only a certain degree of patchiness is necessary, and with the usually applied quadrat sizes up to 25 $\mathrm{dm}^{2}$, probably in most vegetations this condition is fulfilled. Care should be taken in applying the DWR method for estimating species composition in recently sown grasslands where species usually occur more or less at random. In those cases, in principle a very small sampling quadrat (smaller than $1 \mathrm{dm}^{2}$ ) could be used. However, this has practical limitations since the quadrat size should not be too small for realistic yield estimations, needed for the Haydock \& Shaw yield correction.

The simulations revealed that one condition (i.e., that the sampling quadrat should be at least as large that it usually contains three or more species) is not necessary because of the almost always perfect functioning of the correction for missing ranks ('t Mannetje \& Haydock, 1963). Generally speaking, a sampling quadrat should be chosen not larger than is strictly necessary from the viewpoint of horizontal vegetation structure and from the viewpoint of realistic yield estimations.

Multipliers calculated from simulation data could satisfactorily mimic the original multipli-
\end{abstract}


ers of DWR given by 't Mannetje \& Haydock (1963). It is postulated that the DWR method is well suited for studying vegetation changes in old, floristically diverse grasslands with dominant species often in moderate dry weight proportions and species usually growing in patches.

Keywords: dry weight rank, grassland, sampling, simulation, botanical composition, dry weight proportion, presence frequency, rank proportion.

\section{Introduction}

The Dry Weight Rank (DWR) method for the analysis of botanical composition of pastures was developed in Australia by 't Mannetje \& Haydock (1963) to estimate quickly and accurately the species composition of grassland swards on a dry weight basis. The only methods available earlier were either hand-sorting of cut samples (labour intensive) or estimates by eye (not reliable). The DWR method calculates for each species its dry weight proportion $\left(D W_{A} \%\right.$ for species $A$ ) from the percentages of cases it takes the first $(A 1 \%)$, second $(A 2 \%)$ and third $(A 3 \%)$ rank in sampling quadrats on the basis of dry weight (visual estimates). These proportions are weighted by the empirical coefficients $0.702,0.211$ and 0.087 , and added according to:

$$
D W_{A} \%=0.702(A 1 \%)+0.211(A 2 \%)+0.087(A 3 \%)
$$

The coefficients were derived by means of linear multiple regression using sets of data from which the exact dry weight proportions of all species were known. They were modified by Jones \& Hargreaves (1979) to 0.714, 0.247 and 0.039 from further sets of data, although according to the authors these new coefficients do not lead to substantially better results.

DWR was developed from the Rank Method of De Vries (De Vries, 1933; De Vries \& De Boer, 1959) and is a fast method, because there is no need of cutting and hand-separating samples. The observer has only to decide whether there is a greater weight of one species than of another. However, the method requires experienced observers and training beforehand is essential. Difficulties may arise because of large possible differences in dry matter content between species, and because some species are more prominent to the eye than others and tend to be overestimated.

One of the restrictions of the method is that the calculated dry weight proportions can never exceed the value of $70.2 \%$. This can be overcome by allocating first and second ranks to any species which occupies at least $85 \%$ of the total dry matter of a quadrat (Jones \& Hargreaves, 1979). Another problem arises when there is a constant relationship between species dominance and quadrat yield. If a particular species always takes first rank in high yielding quadrats and another one always takes first rank in low yielding ones, the former will be underestimated and the latter overestimated. This can be solved by applying the yield correction of Haydock \& Shaw (1975) (see also Jones \& Hargreaves, 1979).

't Mannetje \& Haydock (1963) did not find an effect of quadrat size on the results 
of DWR and claimed that the method is universally applicable regardless the type of vegetation because it is based on dry weights. On the other hand, rank proportions are frequency proportions, while it is known from presence frequency estimates of species in sampling quadrats (Greig-Smith, 1983) that these are strongly influenced by pattern. Van Dyne et al. (1975) questioned whether the DWR method is really independent of vegetation structure.

The possible role of quadrat size and pattern was investigated by means of a statistical model by Sandland et al. (1982). They studied the role of quadrat size by varying the total plant numbers in quadrats in samplings of fictitious mixtures with Poisson-distributed species (i.e., all species have Poisson-distributed plants), and concluded that samplings with large quadrats theoretically lead to heavy overestimations of high dry weight proportions. Clumping of species, studied using special statistical functions, seemed to improve the DWR estimations in principle. However, the authors also stated that further quantification of these effects in a purely statistical way would be very difficult.

In this paper the effect of quadrat size and clumping on DWR estimations is analysed by means of a computer sampling program. The effect of quadrat size was analysed for randomly distributed species in the same way as in the simulations by Sandland et al. (1982) through varying the total plant numbers per quadrat. The influence of the pattern of plant distribution of species was analysed in a more simple way, namely by allotting species to randomly distributed circular patches. Finally, the values of the multipliers in the DWR equation and the implications of this study for field sampling are discussed.

In principle, the effect of species differences in plant size or dry matter content on DWR estimations can also be studied with the model, but for sake of clearity the presented results in this paper refer to the situation where all species have the same area and yield per plant.

\section{Theory and procedure of simulation}

\section{Assumptions in the calculations}

As in field sampling, a decision has to be made whether the ranks of plant species in sampling quadrats are determined by whole plants falling within the quadrat with their rooted centre, or by all plant parts falling within a vertical column above the quadrat, irrespective whether these parts belong to plants rooting inside or outside the quadrat. In practice, DWR is generally carried out according to the second method. In that case there is also less chance that in individual quadrats not all three ranks are occupied. In our model we followed this second method.

In all calculations all species are assumed to have circular plants of same size, the same yield per plant and the same dry matter content. This implies that their dry weight proportions are identical to their plant number proportions. 


\section{Sampling}

General procedure. Main element of the simulation model is a computer sampling program with which a fictitious vegetation (mixture) can be sampled with a circular sampling quadrat. For that purpose $n$ fictitious square areas are created on which the sampling quadrat is placed; $n$ can be considered as the number of observations (samplings). The numbers of plants in the single squares are determined by drawing a number from an array (ARRAY-1), in which all the theoretically possible plant numbers at the given mean number $(\mathrm{z})$ of plants per square are present in accordance with their probability. The plants are distributed over the square areas by randomly drawing $x$ - and $y$-coordinates from another array (ARRAY-2). The plants are also assigned a plant size (radius $\mathrm{r}$ ). From their $\mathrm{x}$ - and $\mathrm{y}$-coordinates and radii, and the $\mathrm{x}$ and $y$-coordinates (centre point) and radius $(\mathrm{R})$ of the sampling quadrat it is calculated whether plants fall within the quadrat or are only so much as touched by the quadrat, and thus whether they are to be recorded as present.

Species composition is treated in the program by drawing for each plant recorded as present, a species name from a third array (ARRAY-3), in which each species is represented in a frequency that agrees with its dry weight proportion in the whole mixture. These dry weight proportions of the species are calculated from a geometric series relationship (see below).

In the default version of the sampling program all species are assumed to have Poisson-distributed plants (i.e. random distribution). To study the effect of clumping, species growing in patches are simulated with a modified version of the model by projecting circular patches in the squares in a given density. For reasons of simplicity the restriction has been built-in that within one patch only one species can occur. To this end, species names are drawn from ARRAY-3 not for single plants but for groups of same plants in patches. Also patches are allotted $\mathrm{x}$ - and $\mathrm{y}$-coordinates drawn from an array (ARRAY-2) and a sequential number. For plants in the overlap zones of patches, always the species name assigned to the patch with the higher patch number counts.

From the plant numbers per species recorded as present in the quadrat, the rank of each species is determined. When one or more species have the same number of plants (= same dry matter yield) in a quadrat, each species is allocated the same share of the relevant rank. Thus when two species are equal in first and second rank those species receive 0.5 of rank 1 and of rank 2 . When three species are equal in rank they each receive 0.333 of ranks 1,2 and 3 . After sampling of the last square, the ranks of the species are summed in order to calculate their total proportions of first, second and third ranks.

Probability of plant numbers in single samples. The mean number $(z)$ of plants in the squares is calculated from the mean total plant density ( $n$ plants $\mathrm{dm}^{-2}$ ) of the fictitious mixture and the area $\left(\right.$ Area; $\left.\mathrm{dm}^{2}\right)$ of the squares:

$$
z=n * \text { Area }
$$


From $z$ the probabilities $(p(i))$ of all theoretically possible plant numbers $(i)$ in the single samples are calculated until their sum equals 0.999 . Probability $p(i)$ is calculated from (Poisson):

$$
p(i)=\frac{e^{-z} z^{i}}{i !}(0 \leq p \leq 1)
$$

Calculation of mass proportions from the geometric series. According to the geometric series, the proportional contribution $(P R)$ of a species $(S x)$ can be calculated from its rank $\left(R_{S x}\right)$ in mass and a parameter $k$ (May, 1975; Magurran, 1988):

$$
P R_{S x}=k(1-k)^{R_{S x}-1}
$$

Parameter $k$ gives the fractional reduction in proportion between successively ranked species. From the $k$-values dry weight proportions $\left(D W \%_{S x}=P R_{S x} * 100\right)$ can be calculated in principle for an infinite number of species. However, for reasons of limitations in the simulation program the maximum number of species in the mixtures was usually set at 6 . We solved this limitation by taking in a simulation of $n$ species $(1<n \leq 6)$, the dry weight proportions of the $n^{\text {th }}$ species and higher (species theoretically present but not represented in the mixtures) together as a rest-species. This also implies that for low $k$-values in cases of simulations with only 3 species the summed dry weight proportion for this rest-species can be higher than the dry weight proportion of the third, second or dominant species. Therefore, in ranking the species, the rest-species can get a first, second or third rank in these cases. As follows from Equation $4, k$ always reflects the mass proportion of the dominant species.

Scott (1986) concluded from observations in pastures at Parkhouse (Rothamsted, UK) that the geometric series fitted the dry weight proportions of species well in grasslands. Calculated according to Equation 4, the value of $k$ in one treatment had increased from 0.2 in 1856 (floristically very diverse vegetation) to 0.7 in 1919 when the pasture was dominated by one species due to manuring.

Calculation of the numbers of patches. In all simulations with species in patches, always the dominant species is kept in the background area while all other species are in patches. The latter means that the cover by patches $\left(\right.$ Cover $\left._{\text {patch }}\right)$ equals the total space occupied by these other species. This total space is given by the summed relative plant numbers of these species, and since all species have the same horizontal plant size and the same yield per plant also by their summed dry weight proportions. For randomly (Poisson) distributed patches, the Patch Area Index (PAI) can be calculated from Cover $_{\text {patch }}$ according to:

$$
P A I=-\ln \left(1-\text { Cover }_{\text {patch }}\right)
$$

since

$$
\text { Cover }_{\text {patch }}=1-e^{-P A I}
$$


The mean number of patches per unit area, i.e. patch density $\left(d_{\text {patch }}\right)$ is:

$$
d_{\text {patch }}=P A I /\left(\pi R^{2}\right)
$$

in which $R$ is patch radius.

The mean number of patches $\left(z_{\text {patch }}\right)$ in an area of size $A$ is:

$$
z_{\text {patch }}=d_{\text {patch }} A
$$

\section{Correction for missing ranks}

In cases of missing second and third ranks the calculated dry weight proportions from DWR do not sum up to $100 \%$. This problem is solved by first adding the products of multipliers and rank proportions (Equation 1), to give a score for each species ('t Mannetje \& Haydock, 1963). The ultimate dry weight proportions are obtained by expressing for each species its score as a percentage of the sum of the scores of all species together. Totally empty quadrats may also occur but are left out of the calculation because they do not contribute to the dry weight proportions.

\section{Scale of simulation}

Where possible, data used in the simulated samplings are on field scale. All simulations are executed with a plant density of 3 plants $\mathrm{dm}^{-2}$. This density originates from plant density experiments of Kreuz (1969) and Van Loo (1991) with Lolium perenne and could be considered as the equilibrium density of a Lolium perenne sward 3 years after sowing (Neuteboom et al., 1992). The patch sizes used in the simulations for species in patches are in between the sizes found for dung and urine patches in the literature. Richards \& Wolton (1976) calculated a mean dung patch size of $5 \mathrm{dm}^{2}$ and a mean size of urine patches of $49 \mathrm{dm}^{2}$. Dung and urine patches and also mole patches are often the causes of the clumped occurrence of species in grassland (Wind et al., 1993). Below we will show that the maximum patch size of $13.8 \mathrm{dm}^{2}$ used in the simulated samplings was large enough to demonstrate the effect of patchwise distributed species in DWR sampling. In all simulations all species were assigned a plant radius of $1 \mathrm{~cm}$ and were assumed to have the same growth habit and tiller morphology.

Theoretical samplings were executed for different cases with always series of 19 or more diverging mixtures containing 3 or 6 species. The number of samplings per mixture was set at 50 or 80 . The diverging dry weight proportions of the species were obtained according to the geometric series using incremental ranges of $k$-values (Equation 4). First, samplings with different quadrat sizes $(0.176,1.662$ and 8.786 $\mathrm{dm}^{2}$ ) were carried out in series of mixtures with Poisson-distributed species. Next, samplings were done with the large quadrat size of $8.786 \mathrm{dm}^{2}$ in mixtures with species in patches with areas of $2.54 \mathrm{dm}^{2}$ (patch radius $0.9 \mathrm{dm}$ ), $4.52 \mathrm{dm}^{2}$ (patch radius $1.2 \mathrm{dm}$ ) and $13.85 \mathrm{dm}^{2}$ (patch radius $2.1 \mathrm{dm}$ ), respectively. 


\section{Results}

\section{Effects of quadrat size for species growing at random}

Large quadrats. In Figure 1 an example of a DWR sampling of mixtures with Poisson-distributed species with a large sampling quadrat of $8.786 \mathrm{dm}^{2}$ is given. The sampling data are summarised in Table 1. In Figure 1a, the first, second and third rank proportions of the species are plotted against their real whole mixture dry weight proportions $(3$ species $* 24$ mixtures $=72$ points per rank; each point was obtained from 80 samples, i.e. 80 samples were taken per mixture). In Figure 1c, infor-
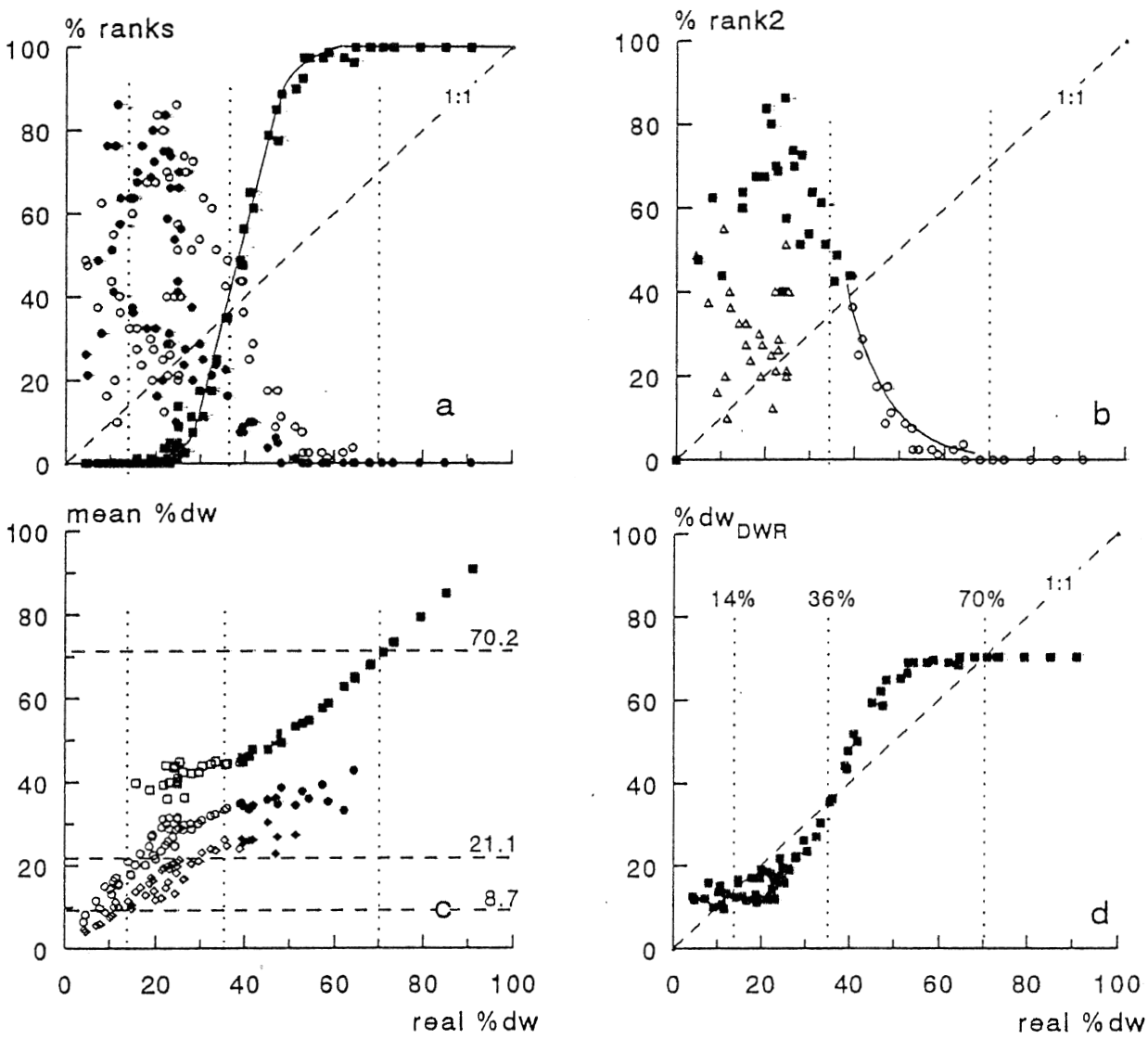

Figure 1. Output of samplings of mixtures of 3 species with a large sampling quadrat of $8.786 \mathrm{dm}^{2}$. The species are randomly distributed. Plotted against the real whole mixture dry weight proportions are: (a) all three rank proportions (rank $1(\mathbf{\square})$, rank $2(\bigcirc)$ and rank $3(\bullet)$ ), (b) the second rank proportions $(\%$ rank 2), (c) the actual mean dry weight proportions in single quadrats at which species got first $(\square, \boldsymbol{\square})$, second $(O, \bullet)$ and third $(\diamond, \diamond)$ placings, and (d) the whole mixture dry weight proportions estimated from DWR. In (b) the three species are distinguished by different markers. In (c) different markers have been used for the dominant species (closed symbols) and the remaining second and third species (open symbols). 
J.H. NEUTEBOOM, E.A. LANTINGA AND P.C. STRUIK

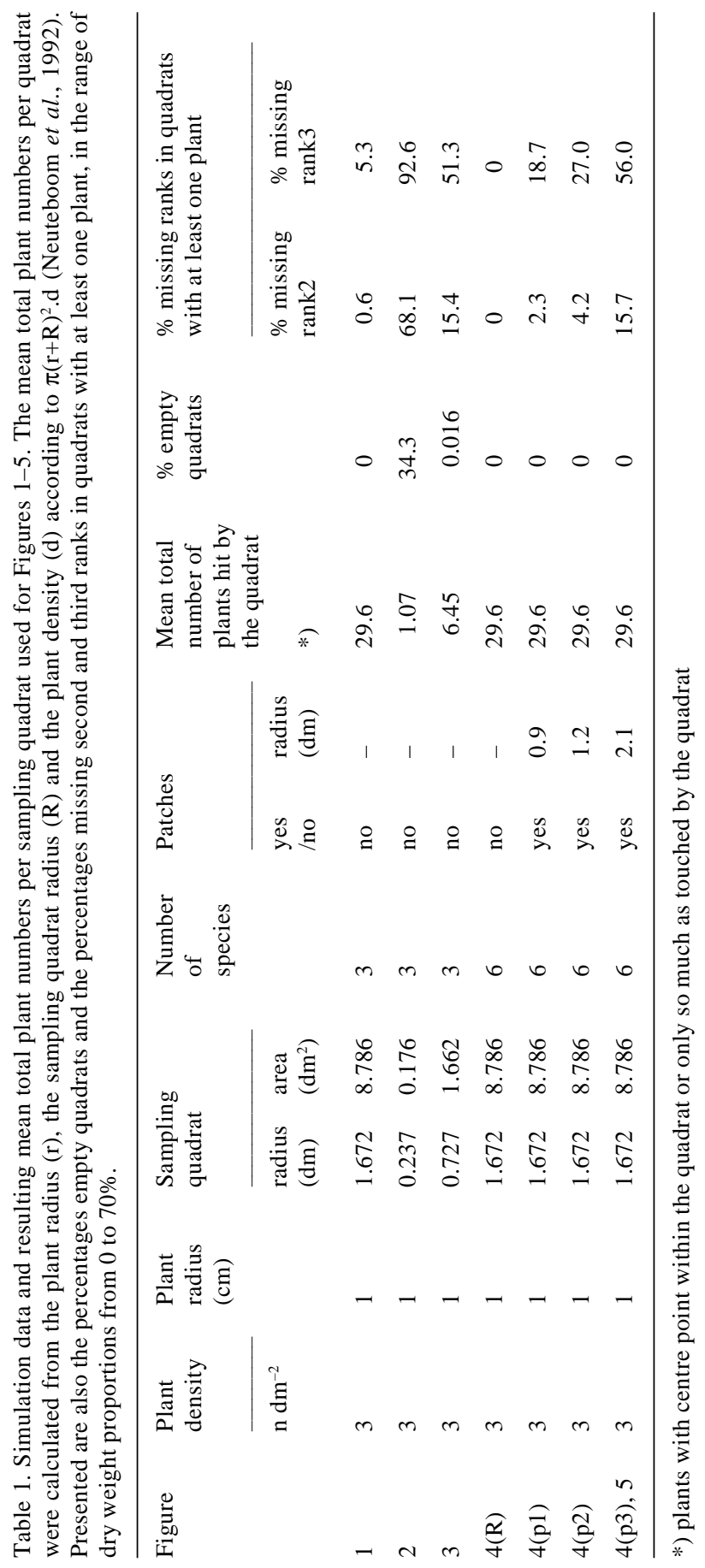


mation is given on the mean dry weight proportions in single quadrats at which species obtained first, second or third ranks. Note that the dominant species and the remaining second and third species in the mixtures have been given different markers. This figure clearly shows that the mean dry weight proportions at which species got first, second and third placings in single quadrats differed for diverging mixture compositions and also for the dominant, second and third species in the mixtures. These mean dry weight proportions can be compared with the coefficients of DWR which assign to these ranks dry weight proportions of $70.2 \%, 21.1 \%$ and $8.7 \%$, respectively. Figure $1 \mathrm{~d}$ shows for all species their whole mixture dry weight proportions estimated from DWR, in a plotting against their real whole mixture dry weight proportions.

Species are especially overestimated by DWR in the range of real dry weight proportions between 36 and $70 \%$ (Figure 1d) due to both the dominant occupation of first ranks (even at only moderately high dry weight proportions) by the dominant species (Figure 1a), and the high first coefficient of DWR (Figure 1c). In the range of dry weight proportions below $36 \%$ where species mainly obtained second and third ranks (Figures 1a and b), they first (from about 14\% to 36\%) were assigned too little and then (below about 14\%) too much of the second and third coefficient of DWR (Figure 1c).

The strong variation in the second and third rank proportions at the same real whole mixture dry weight proportions (Figure 1a) is not only caused by sampling errors. Part of the variation is due to the fact that each species has to compete with other species for its ranks. It makes a difference whether in the fictitious case of a species with $30 \%$ of total dry weight in a 3 -species mixture, the companion species have dry weight proportions of e.g. $65 \%$ and $5 \%$, or $45 \%$ and $25 \%$, respectively. In the first case, the $30 \%$-species will have almost all second ranks and only very few third ranks, while in the second case it will have second and third rank proportions more similar to those of the other two species. This possibly strong variation in second and third rank proportions at the same real whole mixture dry weight proportions is illustrated for the second rank proportions in Figure 1b where different markers for the three species have been used.

Small quadrats. Figures 2a-d illustrate for series of mixtures of 3 species, the extreme case of a DWR sampling with a very small sampling quadrat of $0.176 \mathrm{dm}^{2}$ (sampling data given in Table 1). Due to the low mean total plant number per quadrat of 1.07, and the small plant radius of $1 \mathrm{~cm}$, many sampling quadrats were empty; the presence frequency $(P \%)$ of plants is $65.7 \%(P \%$ at $\% \mathrm{dw}=100$ in Figure 2a; curve 1). However, since empty quadrats do not contribute to the dry weight proportions, they can be left out from the calculations. To this end, the rank proportions for the first, second and third ranks are recalculated by multiplying them by a factor $100 / 65.7$ (Figure 2b). The curve for the percentages first ranks coincides almost with the $45^{\circ}$ line now, and will be identical to this line, together with the corrected curve for $P \%$ (curve 2 in Figure 2a), when both plant size and quadrat size are reduced to a point. In that case there will be no second and third ranks anymore, because due to the very low mean plant numbers per quadrat not more than only one plant will be 

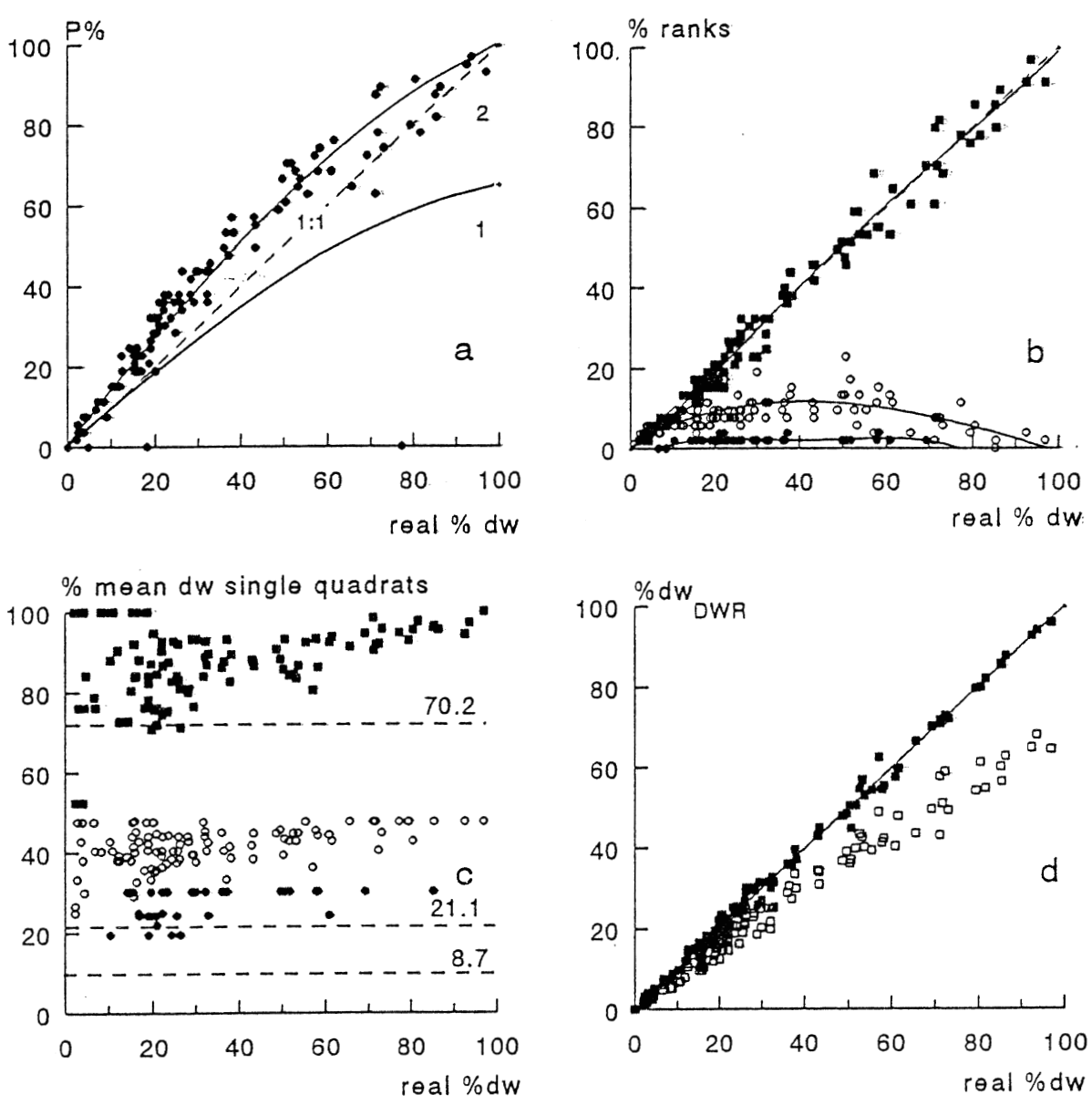

Figure 2. Output of samplings of mixtures of 3 species with a small sampling quadrat of $0.176 \mathrm{dm}^{2}$. The species are randomly distributed. Plotted against the real whole mixture dry weight proportions of the species are: (a) the presence frequency in the quadrats (curve 1) and the presence frequency corrected for empty quadrats (curve 2), (b) the three rank proportions corrected for empty quadrats (rank 1 ( $\mathbf{\square}$ ), rank $2(\bigcirc)$ and rank $3(\bullet)$ ), (c) the actual mean dry weight proportions in single quadrats at which species got first $(\boldsymbol{\square})$, second $(\mathrm{O})$ and third $(\mathbf{O})$ placings, and $(\mathrm{d})$ the whole mixture dry weight proportions estimated from DWR, corrected $(\boldsymbol{\square})$, and not corrected $(\square)$ for missing second and third ranks.

hit per quadrat. The equation of 't Mannetje \& Haydock (1963) based on proportions would lead to a maximum attainable dry weight proportion of only $70 \%$, and thus, to strong underestimations (the open symbols in Figure 2d). However, all the component species are underestimated by DWR to more or less the same extent over the whole range of real whole mixture dry weight proportions (Figure 2c), and therefore almost perfect DWR estimations were obtained after correction for missing second and third ranks (the closed symbols in Figure 2d). 
Medium quadrats. Sampling of series of 3-species mixtures with a medium quadrat of $1.662 \mathrm{dm}^{2}$ (sampling data given in Table 1) results in a mean plant number per quadrat of 6.45. There are hardly empty quadrats but still considerable percentages missing second and third ranks. Also this simulation yielded a curve for the percentages first ranks closer to the $45^{\circ}$ line than with the large quadrat (compare Figure $3 \mathrm{a}$ with Figures $1 \mathrm{a}$ and $2 \mathrm{~b}$ ) and gave satisfactory estimations of dry weight proportions with the DWR calculation after correction for missing ranks (Figure $3 b$ ).

Also with this quadrat size, the mean dry weight percentages at which species obtain first second and third ranks in single quadrats were totally different from the $70.2 \%, 21.1 \%$ and $8.7 \%$ suggested by the DWR multipliers (Figure 3c). One of the reasons that the multipliers together can still result in acceptable estimations of dry weight proportions is, that in conjunction with the rank proportions, overestimations by the first multiplier are levelled out to a large extent in the range of real dry weight proportions between about $10 \%$ and $60 \%$ by underestimations through the second and third multipliers.
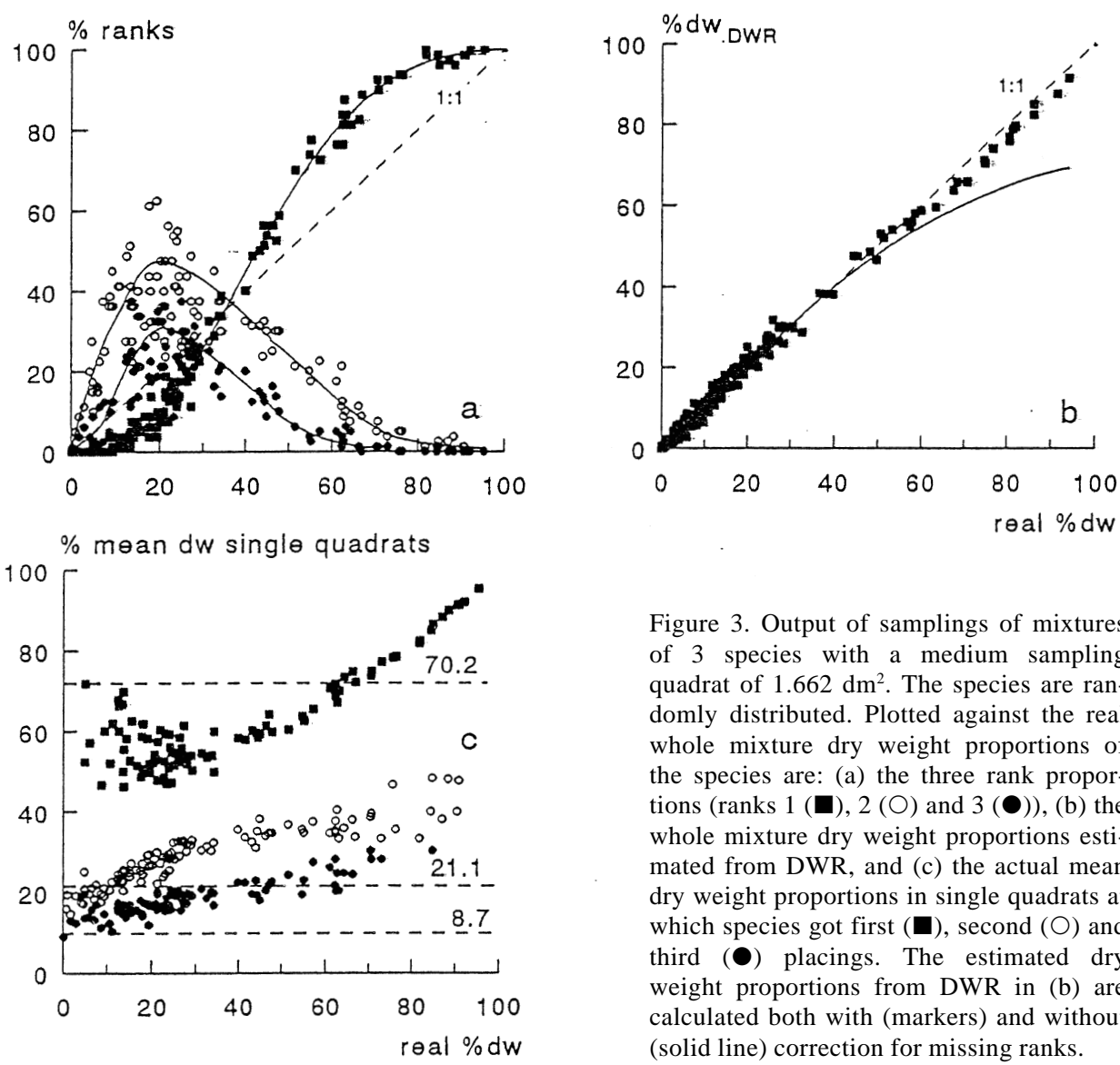

Figure 3. Output of samplings of mixtures of 3 species with a medium sampling quadrat of $1.662 \mathrm{dm}^{2}$. The species are randomly distributed. Plotted against the real whole mixture dry weight proportions of the species are: (a) the three rank proportions (ranks $1(\mathbf{\square}), 2(\bigcirc)$ and $3(\bullet)$ ), (b) the whole mixture dry weight proportions estimated from DWR, and (c) the actual mean dry weight proportions in single quadrats at which species got first $(\mathbf{\square})$, second $(O)$ and third (-) placings. The estimated dry weight proportions from DWR in (b) are calculated both with (markers) and without (solid line) correction for missing ranks. 


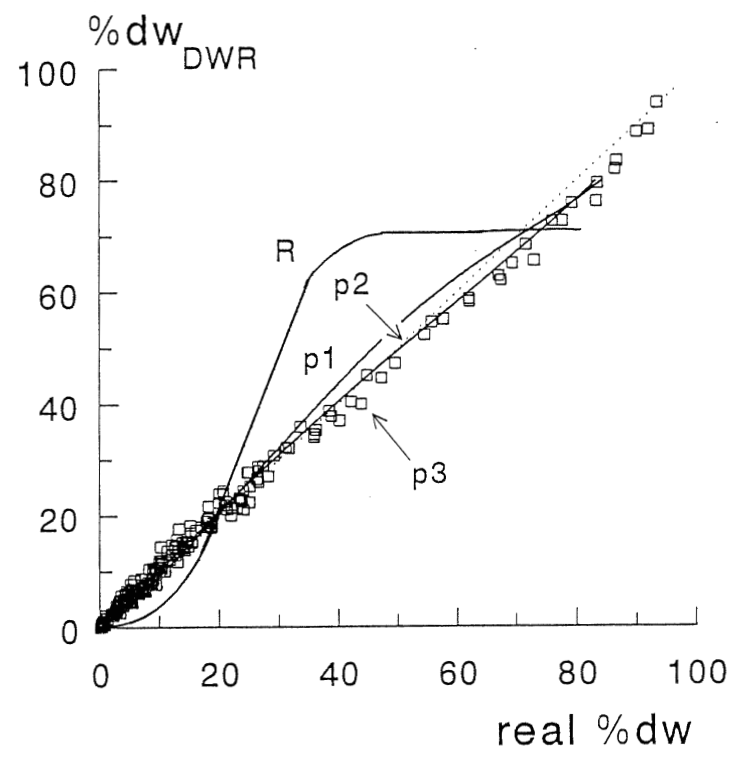

Figure 4. Output of samplings of mixtures of 6 species using a large sampling quadrat of $8.786 \mathrm{dm}^{2}$. There are 4 cases: species randomly distributed $(\mathrm{R})$, and species in patches of $2.54 \mathrm{dm}^{2}$ (patch radius $0.9 \mathrm{dm}$; p1), $4.52 \mathrm{dm}^{2}$ (radius 1.2 $\mathrm{dm}$; p2) and $13.8 \mathrm{dm}^{2}$ (radius $2.1 \mathrm{dm}$; p3), respectively. Plotted against each other are the estimated dry weight proportions from DWR and the real whole mixture dry weight proportions. For 3 cases $(\mathrm{R}, \mathrm{p} 1$ and $\mathrm{p} 2)$ the relation between both parameters is represented by a smoothed line; for one case (p3) the single points have been plotted. The DWR estimated dry weight proportions were corrected for missing ranks.

\section{Effect of plant distribution of species}

Figure 4 summarises the final output from samplings with a large quadrat of 8.786 $\mathrm{dm}^{2}$ in 6-species mixtures allotted to patches; patches of $2.54(\mathrm{p} 1), 4.52(\mathrm{p} 2)$ and $13.8(\mathrm{p} 3) \mathrm{dm}^{2}$, respectively. Curve $\mathrm{R}$ in the graph is for comparison and resulted from sampling with the same quadrat size in mixtures with species distributed randomly. The data for the largest patch size sampling are presented in more detail in Figures 5a-d.

Figures 4 and 5a-d clearly show that samplings with a large quadrat in mixtures with species in patches have more or less the same result as samplings with a small or medium quadrat in mixtures with randomly distributed species (compare with Figures 2 and 3 ). It can be concluded that species growing in patches lead to a more straightforward curve for first rank proportions using the same large quadrat size (compare Figure 5a with Figure 1a). As in Figures 1c, 2c and 3c, the actual mean dry weight proportions at which species obtained first, second and third ranks in single quadrats were again different from what is suggested by the DWR coefficients (Figure 5c). Compared with the random distribution of species (Figure 4, curve R) satisfactory DWR estimations were already obtained with species assigned to the relatively small patch size of $2.54 \mathrm{dm}^{2}$ (Figure 4, p1). The larger patch sizes $4.52 \mathrm{dm}^{2}$ (Figure 4, p2) and $13.8 \mathrm{dm}^{2}$ (Figure 4, p3) did not substantially improve the estimations.

\section{Calculation of DWR coefficients from simulation}

From the data in Figures 4 and 5 (6-species mixtures and a large sampling quadrat of $8.786 \mathrm{dm}^{2}$ ), DWR coefficients were calculated from linear multiple regression 

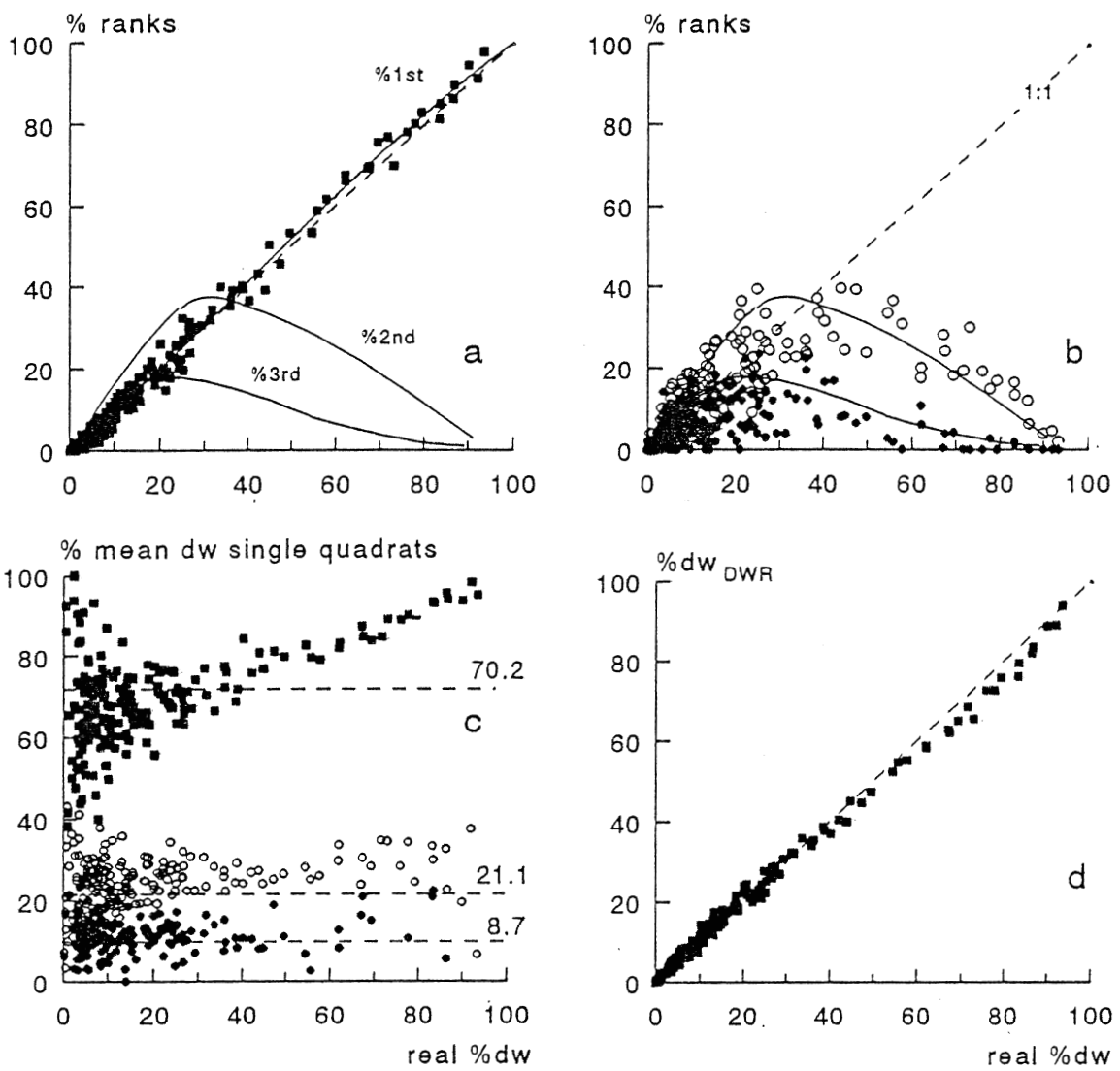

Figure 5. Detailed output of the samplings of mixtures of 6 species with species in patches of $13.8 \mathrm{dm}^{2}$ (patch radius $2.1 \mathrm{dm}$ ) using a large sampling quadrat of $8.786 \mathrm{dm}^{2}$. Plotted against the whole mixture dry weight proportions of the species are: $(\mathrm{a}, \mathrm{b})$ the three rank proportions $(\mathbf{\square} ; \% 1 \mathrm{st}),(\bigcirc ; \% 2 \mathrm{nd}),(\boldsymbol{\bullet}$ $\% 3 r d)$; (c) the actual mean dry weight proportions in single quadrats at which species got first ( $\mathbf{\square})$, second $(O)$ and third $(\bullet)$ placings, and, (d) the whole mixture dry weight proportions estimated from DWR and corrected for missing ranks.

Table 2. DWR coefficients calculated from regression using simulated data in the range of dry weight proportions from 0 to 70\%, and those given by 't Mannetje \& Haydock (1963; DWR1) and Jones \& Hargreaves (1979; DWR2).

\begin{tabular}{llllll}
\hline Figure & Patch radius $(\mathrm{dm})$ & \multicolumn{2}{l}{ Coefficients } & $\mathrm{R}^{2}$ \\
\cline { 3 - 5 } & & Rank 1 & Rank 2 & Rank 3 & \\
& & & & \\
$4(\mathrm{R})$ & random & 0.569 & 0.204 & 0.227 & 0.956 \\
$4(\mathrm{p} 1)$ & 0.9 & 0.676 & 0.205 & 0.119 & 0.989 \\
$4(\mathrm{p} 2)$ & 1.2 & 0.765 & 0.208 & 0.027 & 0.987 \\
$4(\mathrm{p} 3), 5$ & 2.1 & 0.851 & 0.186 & -0.037 & 0.990 \\
& & & & & - \\
DWR1 & - & 0.702 & 0.211 & 0.087 & - \\
DWR2 & - & 0.714 & 0.247 & 0.039 & - \\
\hline
\end{tabular}


through the origin in the same way as done by 't Mannetje \& Haydock (1963). Regressions were executed for the range of dry weight proportions from 0 to $70 \%$ (Table 2). Especially the coefficients for the patch radii 0.9 and $1.2 \mathrm{dm}$ were close to the DWR coefficients of 't Mannetje \& Haydock (1963) and Jones \& Hargreaves (1979). The values of the coefficients obtained will depend on the range of the mixture dry weight proportions included in the regression and on the distribution of the dry weight proportions within that range. The better the correlation between the dry weight proportions and first rank proportions, the higher will be the coefficient for the first ranks. This explains the high first coefficient found for the simulation with the large patch radius of $2.1 \mathrm{dm}$. In that situation a negative coefficient was found for the third rank proportions.

\section{Validation from field data}

In Figures 6a-d field data are presented from a botanical study of 37 Dutch grasslands by Dirven \& Wind (1982). They estimated first, second and third rank proportions of plant species in $0.25 \mathrm{dm}^{2}$ cores (100 cores per field) and determined their dry weight proportions by hand separation of subsamples (one subsample per field). In the figures only the data of Lolium perenne and Poa trivialis are presented.

The rank-\%dw relationships (Figures 6b-d) support the theoretically calculated curves (see e.g. Figure 5a). However, there is still some scatter in the relation between the estimated and the real dry weight proportions (Figure 6e). Moreover, there is a slight but systematic underestimation of the dry weight proportions of $L$. perenne and a slight but systematic overestimation of $P$. trivialis. The calculated dry weight proportions were not corrected for missing ranks and, unfortunately, also the Haydock \& Shaw yield correction (Jones \& Hargreaves, 1979) was not carried out.

Part of the scatter might have been due to sampling errors since a very small sampling quadrat was used. The reason for the respective under- and overestimations of $L$. perenne and $P$. trivialis might have been the patchwise occurrence of one or both, or of other component species combined with a difference in their productivity. Jones \& Hargreaves (1979) demonstrated that patches of high and low yielding species can lead to underestimations and overestimations, respectively. L. perenne might have occurred more patchwise in the paddocks than $P$. trivialis, as suggested by Figure 6a where the presence frequencies of both species are plotted against their real dry weight proportions. L. perenne had a lower presence frequency at the same dry weight proportions, and is generally considered as higher yielding than $P$. trivialis (De Vries et al., 1942; Wells \& Haggar, 1974).

\section{Discussion}

\section{Rank proportions}

The results from the simulations and the field study by Dirven \& Wind (1982) show 

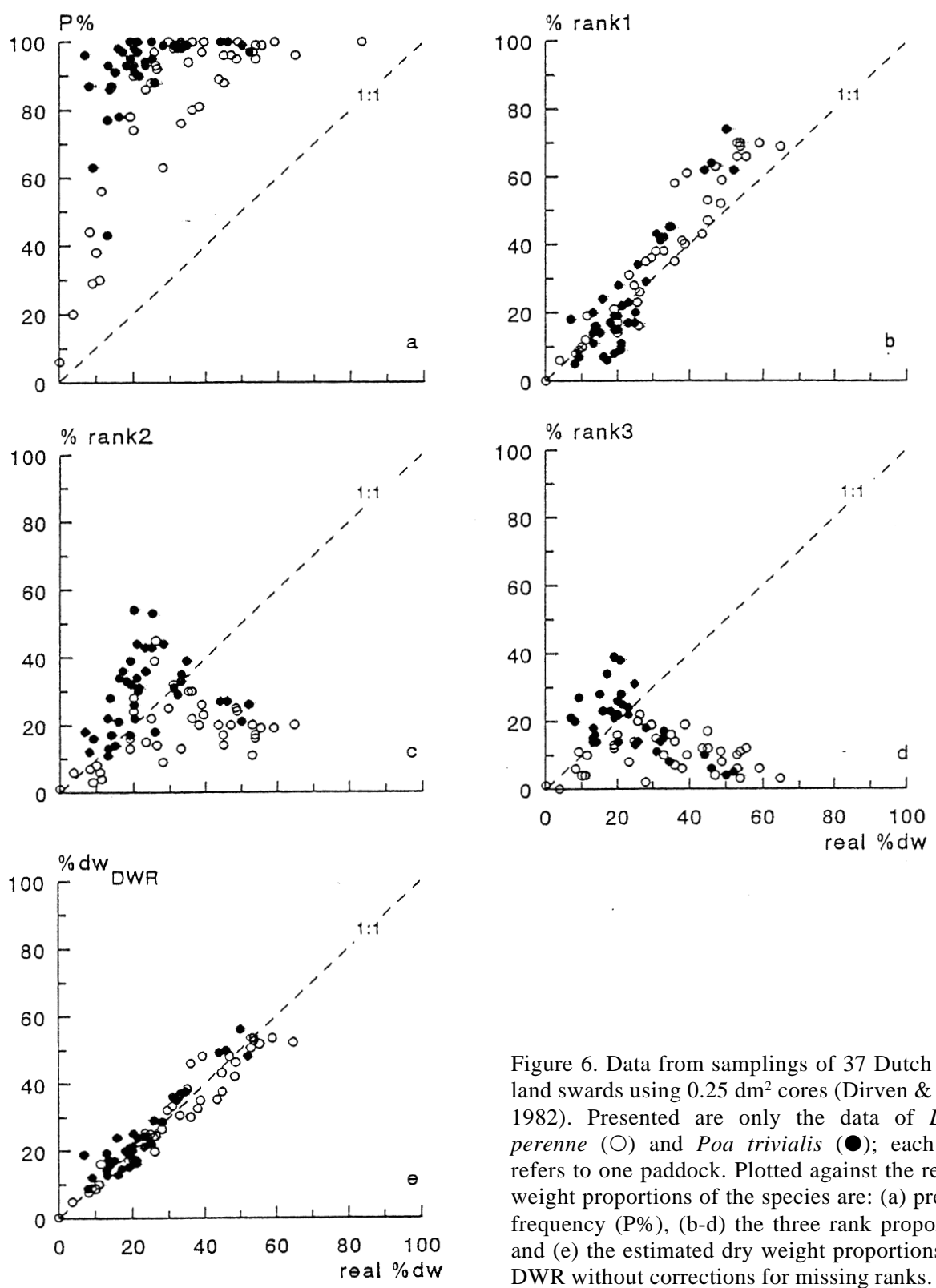

Figure 6. Data from samplings of 37 Dutch grassland swards using $0.25 \mathrm{dm}^{2}$ cores (Dirven \& Wind, 1982). Presented are only the data of Lolium perenne $(\bigcirc)$ and Poa trivialis $(\bullet)$; each point refers to one paddock. Plotted against the real dry weight proportions of the species are: (a) presence frequency $(\mathrm{P} \%)$, (b-d) the three rank proportions, and (e) the estimated dry weight proportions from DWR without corrections for missing ranks.

that the rank-abundance relationships on which the DWR method is based are not linear. In plottings against their real whole mixture dry weight proportions, the first rank proportions show an $\mathrm{S}$-shaped curve and the second and third rank proportions show single-peaked curves (e.g. Figure $3 a)$. The rank proportions are mutually dependent, which explains why the second and third rank proportions decline to zero 
when the first rank proportion increases to $100 \%$. This mutual dependence of the dry weight proportions could be one of the reasons, why in spite of the curved relationships, a linear multiple regression through the origin of dry weight proportions (y) on rank proportions (x) may still work satisfactorily.

\section{Effects of quadrat size and plant distribution of species}

Sandland et al. (1982) concluded from their model that in samplings of mixtures with Poisson-distributed species, the use of a large sampling quadrat with high mean plant numbers per quadrat (set at 50), can lead to a large overestimation of the dry weight proportions of the dominant species. The same was concluded from our model where we also found that in this situation the species of low dry weight proportions are sometimes underestimated (Figure 1). The over- and underestimations are due to the relatively small variation in plant numbers of the species in large quadrats using the Poisson function together with the high first coefficient of DWR. In the extreme case of species with Poisson-distributed plants, large quadrats each reflect the species composition of the whole mixture, which means that if a species is dominant in the whole mixture at for example 50\%, it will be dominant in almost all sampling quadrats and thus occupy nearly all first ranks at dry weight proportions of approximately 50\%. This results in a strong overestimation of the dry weight proportion of that species by DWR because it is assigned too much for its first ranks from the first coefficient. In Figure 1d the strong overestimation of high dry weight proportions above $36 \%$ was due to the dominant occupation of first ranks by the dominant species (Figure 1a) and overestimation of its real dry weight proportion in single quadrats by the high first coefficient of DWR (Figure 1c) already in a range of only moderately high dry weight proportions. The underestimation of low dry weight proportions in the range between $14 \%$ and $36 \%$ in Figure 1d was due to the fact that the remaining species obtained predominantly second and third ranks at dry weight proportions in single quadrats which were underestimated by the second and third coefficients of DWR.

Good DWR estimations were obtained in sampling mixtures with a relatively small sampling quadrat (smaller than $2 \mathrm{dm}^{2}$ ) containing few plants per quadrat (Figures 2 and 3), or with a large sampling quadrat $\left(8.876 \mathrm{dm}^{2}\right)$ when species were allotted to patches (Figures 4 and 5). Small quadrats lead to better results in mixtures with Poisson-distributed species because with few plants per quadrat also minor species can compete successfully for first, second and third ranks (Figure 2). This can be easily imagined for the extreme case of small quadrats with on average one plant, where, if present, even a rare species can be dominant. The reason why a sampling with large quadrats in mixtures with species in patches also leads to good DWR estimations is similar, because also the clumped occurrence in patches is a way for minor species to compete successfully for first, second and third ranks in single quadrats (Figure 5).

The role of the DWR-coefficients varies in these cases. In the extreme case of very small quadrats and such low plant densities and small plant sizes that plants do not overlap (approximately the case in Figure 2), their values are unimportant be- 
cause only first rank proportions are obtained. Hence, due to the perfect functioning of the correction for missing ranks, the coefficients can be given any value. The same will occur in the extreme case of a sampling with large quadrats in mixtures in which all species grow in mosaic-like large monoculture patches. In both cases the first rank proportions of the species will be linearly correlated with their plant densities, which means that the relation between the first rank proportions and the dry weight proportions is a straight line. In samplings with medium quadrats with relatively low mean plant numbers per quadrat (the case with Poisson-distributed species in Figure 3), and in samplings with large quadrats in mixtures with species growing in relatively small patches (not shown), along with a more pronounced S-shaped curve for first rank proportions, higher second and third rank proportions will occur. This increases the weight of the second and third coefficients, and consequently for all three coefficients also the effects of their over- and underestimations. Two things can happen then: (1) all three coefficients overestimate the real dry weight proportions in single quadrats for the respective ranks they stand for (Figure 2), or (2) the under- and overestimations by the first coefficient go along with over- and underestimations by the second and third coefficients, respectively (Figure 3). Both cases can result into satisfactory DWR estimations. The reason in case (1) is, that if the real dry weight proportions at first, second and third placings are overestimated by the respective coefficients to more or less the same extent (which was approximately the case), these effects are levelled out by the way of calculation of the correction for missing ranks (i.e., all whole mixture dry weight proportions of the species are recalculated after they are summed to $100 \%$ ). In case (2) the over- and underestimations by the first coefficient are levelled out by the under- and overestimations by the second and third coefficient, respectively. These compensating effects can explain why already with relatively small patches (Figure 4; p1 with patch radius $0.9 \mathrm{dm}$ ) very satisfactory DWR-estimations of dry weight proportions were obtained. Larger patch sizes cause a change-over to the levelling-out effects described for case (1) and could lead to higher proportions of missing ranks (Table 1). However, also this effect is successfully levelled out by the correction for missing ranks. Our simulations therefore seem to indicate that although the clumped occurrence of species in patterns is a condition for the proper functioning of DWR in relatively large quadrats, only a certain degree of pattern is necessary.

\section{Which quadrat size should be used?}

Since also plant density and plant size have an influence on the measured effect of quadrat size in DWR, and as these can differ for each situation, we may not directly compare our data from simulation with those from field samplings. Nevertheless, the principle that only a certain degree of patchiness is required for the good functioning of the DWR method could explain why 't Mannetje \& Haydock (1963) and Barnes et al. (1982) did not find an effect of quadrat size. 't Mannetje \& Haydock (1963) compared quadrat sizes in the range from 1 to $25 \mathrm{dm}^{2}$, while Barnes et al. (1982) compared the quadrat sizes $2.25,4$ and $6.25 \mathrm{dm}^{2}$. We also tested a range of quadrat sizes in a DWR field sampling $\left(0.25,1\right.$ and $\left.4 \mathrm{dm}^{2}\right)$ and found no clear effect 
(unpublished data). In conclusion, within the range of quadrat sizes up to $25 \mathrm{dm}^{2}$, probably in most established swards the condition of presence of at least a certain degree of pattern for DWR sampling will be fulfilled. This knowledge may remove the theoretical objections against the DWR method to a great extent.

The question which quadrat size should be used in DWR sampling is also dependent on the required size for realistic yield estimations in order to apply the Haydock \& Shaw (1975) yield correction. Besides, practical considerations play a role such as the applicability of the quadrat with regard to the occurring plant sizes and plant densities. However, the simulations revealed that the condition that the sampling quadrat should be at least as large that it usually contains at least three species ('t Mannetje \& Haydock, 1963) is not necessary because of the almost always perfect functioning of the correction for missing ranks (Figure 2). This is also to be expected from a theoretical point of view, and even the reverse could be stated, namely that the quadrat size should preferably be as small as possible. First, because with decreasing quadrat size the dry weight proportions are becoming better linearly correlated with the first rank proportions, and secondly, because in the outmost case, as was explained before, only first ranks remain which can be given any possible coefficient because of the almost always perfect functioning of the correction for missing ranks. However, very small quadrats would also lead to more empty quadrats which do not give information. Generally speaking, a sampling quadrat should be chosen not larger than is strictly necessary from the viewpoint of horizontal vegetation structure and from the viewpoint of realistic yield estimations.

\section{DWR coefficients}

The DWR coefficients we calculated from sampling (Table 2) were in two cases (the mixtures with patch radii of 0.9 and $1.2 \mathrm{dm}$ ) close to the coefficients of 't Mannetje \& Haydock (1963) and Jones \& Hargreaves (1979). In general, except in the case of random species distributions, the original DWR coefficients by 't Mannetje \& Haydock (1963) proved to work very satisfactorily. New regression coefficients from field data with exact dry weight proportions obtained from hand separation were also calculated by Barnes et al. (1982). Like Jones \& Hargreaves (1979) they found for particular cases sometimes negative coefficients for second or third rank proportions. We also found in one case a negative coefficient. The possibility of finding negative coefficients is inherent to multiple regression. Jones \& Hargreaves (1979) considered the negative multipliers logically absurd, and as these hardly improved the DWR-estimations, they advised against the calculation of multipliers for particular cases.

\section{Effect of number of species}

Close examination of Figures 1, 2 and 3 (three species) and Figures 4 and 5 (six species) shows that in principle also the number of species in the mixture plays a role in the ranges of over- and underestimations of dry weight proportions by DWR. With more species in the mixture the range of overestimations is broader at the ex- 
pense of the range of underestimations. However, this only seems relevant in samplings with large quadrats in mixtures with randomly distributed species where overand underestimations actually can occur, i.e. in recently sown grasslands. In practice in such grasslands the dominance of the sown species is often so strong that already for that reason the DWR method is not the most appropriate one to be used. Field sampling and hand separation is then a more accurate method.

\section{Different yielding of plant species}

For sake of clearity we did not study the effect of different yielding of plant species on DWR estimations with the model. However, such differences will result in differences in single quadrat yields, and as was explained for L. perenne and P. trivialis in Figure 6 these will be largely adjusted for by the yield correction of Haydock \& Shaw (1975).

\section{Conclusion}

The main objective of our study was to investigate whether quadrat size and pattern of plant distribution of species influence the functioning of the DWR method. In conclusion, our simulations seem to indicate that possibly only in recently sown grasslands where random plant distributions can occur (Van Loo, 1991) one should be cautious in applying DWR. However, once the condition of a certain degree of patchiness is fulfilled, and probably this is the case in most older grasslands, DWR will function satisfactorily. The DWR method is often used in typical production grasslands for estimating botanical composition of forages on offer. On the basis of the presented theoretical analyses and the validation from field data, we postulate that the method is also well suited for monitoring vegetation changes, especially in old, floristically diverse grasslands with dominant species often in moderate dry weight proportions and species usually growing in patches. In that case the Haydock \& Shaw (1975) yield correction is not advised because not total forage composition but the mean botanical composition in the horizontal plane is important. Moreover, when ecological information is wanted above all, fresh weight proportions as a measure for the space species occupy in the vegetation are of greater interest than dry weight proportions. Possible differences in dry matter content between species can than be ignored. Nevertheless, DWR (or fresh weight rank) estimations can be best executed in early spring or after a cleaning cut when species differences in yield are relatively small. This avoids the problem of estimating mass proportions in tall grass. Moreover, omitting yield estimations allows more freedom in choosing the quadrat size to be used. We experienced that in short grass vegetations a quadrat of $4 \mathrm{dm}^{2}$ is appropriate.

\section{Acknowledgments}

We thank prof. L. 't Mannetje for his stimulating interest in this work, the discussions we have had and his critical reading of the paper. 


\section{J.H. NEUTEBOOM, E.A. LANTINGA AND P.C. STRUIK}

\section{References}

Barnes, D.L., B.H. Beukes \& J.J. Odendaal, 1982. Use of the dry weight rank method of botanical analysis in the Eastern Transvaal Highveld. Proceedings of the Grassland Society South Africa 17: 79-82.

De Vries, D.M, 1933. The Rank Method. An estimation method for botanical grassland research using rank orders (in Dutch). Verslagen Landbouwkundige Onderzoekingen No. 39A: 1-24. The Hague, The Netherlands.

De Vries, D.M. \& Th.A. De Boer, 1959. Methods used in botanical grassland research in the Netherlands and their application. Herbage Abstracts 29: 1-7.

De Vries, D.M., M.L. 't Hart \& A.A. Kruijne, 1942. A rating system of grasslands based on botanical composition (in Dutch). Landbouwkundig Tijdschrift 54: 245-259.

Dirven, J.P.G. \& K.Wind, 1982. Intensification of grassland exploitation and botanical composition of grass swards (in Dutch). Mededelingen van de Vakgroep Landbouwplantenteelt en Graslandkunde No. 65. Wageningen Agricultural University, Wageningen, 24 pp.

Greig-Smith, P., 1983. Quantitative Plant Ecology. Third edition. Blackwell Scientific Publications, Oxford, $359 \mathrm{pp}$.

Haydock, K.P. \& N.H. Shaw, 1975. The comparative yield method for estimating dry matter yield of pasture. Australian Journal of Experimental Agriculture and Animal Husbandry 15: 663-670.

Jones, R.M. \& J.N.G. Hargreaves, 1979. Improvements to the dry weight rank method for measuring botanical composition. Grass and Forage Science 34: 181-189.

Kreuz, E., 1969. Über den Einflusz des Standraumes auf Mortalität und Ertrag bei Gräsern und Kleearten zur Futternutzung. Albrecht Thaer Archiv 13: 197-212.

Magurran, A., 1988. Ecological Diversity and its Measurement. University Press, Cambridge, 179 pp.

Mannetje, L. 't \& K.P. Haydock, 1963. The dry weight rank method for the botanical analysis of pasture. Journal of the British Grassland Society 18: 268-275.

May, R.M., 1975. Patterns of species abundance and diversity. In: M.L. Cody \& J.M. Diamond (Eds.), Ecology and Evolution of Communities. Harvard University Press, Cambridge, pp. 81-120.

Neuteboom, J.H., E.A. Lantinga \& E.N. Van Loo, 1992. The use of frequency estimates in studying sward structure. Grass and Forage Science 47: 358-365.

Richards, I.R. \& Karen M. Wolton, 1976. The spatial distribution of excreta under intensive cattle grazing. Journal of the British Grassland Society 31: 89-92.

Sandland, R.L., J.C. Alexander \& K.P. Haydock, 1982. A statistical assessment of the dry weight rank method of pasture sampling. Grass and Forage Science 37: 263-272.

Scott, D., 1986. Coefficients for the dry weight rank method of botanical analysis of pasture. Grass and Forage Science 41: 319-321.

Van Dyne, G.M., L.J. Bledsoe, J.D. Gustafson, J.H. Hughes \& D.M. Swift, 1975. A study of the dry weight rank method of botanical analysis. Grassland Biome, Ecosystem analysis studies. U.S. International Biological Program. Technical Report, No. 297. Natural Resource Ecology Laboratory, Colorado State University, Fort Collins, 68 pp.

Van Loo, E.N., 1991. Relationships between sward structure, plant density and dry matter yield of perennial ryegrass (Lolium perenne L.). In: Grassland renovation and weed control. Symposium of the European Grassland Federation, Graz, pp. 167-168.

Wells, G.J. \& R.J. Hagger, 1974. Herbage yields of ryegrass swards invaded by Poa species. Journal of the British Grassland Society 29: 109-111.

Wind, K., J.H. Neuteboom \& L.'t Mannetje, 1993. Effect of extensification on yield and botanical composition of grassland on dry sandy soil. In: R.J. Haggar \& S. Peel (Eds.) Grassland Management and Nature Conservation. Occasional Symposium No. 28. British Grassland Society, Reading, UK, pp. $217-222$. 